\title{
Memantine for the Patients with Mild Cognitive Impairment in Parkinson's Disease: A Pharmacological fMRI Study
}

Shoji Kawashima ( $\nabla$ shoji@med.nagoya-cu.ac.jp )

Nagoya City University

Noriyuki Matsukawa

Nagoya City University

RCIP-Nagoya Study Group

Nagoya City University

\section{Research Article}

Keywords: Mild cognitive impairment, Parkinson's disease, dementia, functional MRI, working memory, nback test, memantine, daytime sleepiness, superior frontal gyrus

Posted Date: October 27th, 2021

DOI: https://doi.org/10.21203/rs.3.rs-967500/v1

License: (9) This work is licensed under a Creative Commons Attribution 4.0 International License. Read Full License 


\section{Abstract}

\section{Background:}

Mild cognitive impairment of Parkinson's disease (PD-MCl) represents increased risk of future cognitive decline. The characteristics of PD-MCl are impairments in executive function and visuospatial recognition. The visuospatial n-back test has a merit that it can assess both cognitive domains.

Concerning the treatment of PD-MCl and dementia in PD (PDD), many studies have reported efficacy of cholinesterase inhibitors. Similarly, some studies reported efficacy of memantine for PDD, showing that it improved clinical status or behavioral symptoms. However, therapeutic evidence of memantine for PD$\mathrm{MCl}$ has not been unestablished.

\section{Methods:}

We aimed to investigate whether memantine can alter brain function of the patients with PD-MCl, using functional MRI. In comparison between memantine and placebo, we explored the difference in regions associated with visuospatial n-back test. The 0-back test reflects visuospatial recognition, and the 1-back and 2-back tests reflect visuospatial working memory. This study followed a randomized double-blind crossover design. Patients in the memantine group were given memantine at $5 \mathrm{mg} / \mathrm{day}$ in the first week, and the dose was increased by $5 \mathrm{mg} /$ day per week, with the final dose of $20 \mathrm{mg} /$ day. The patients in the placebo group were given a placebo following the same regimen. The population in this study constitutes 10 patients who completed follow-up. During maximum dose administration, fMRI scanning and neuropsychological tests were performed. Group comparisons between memantine and placebo were performed.

\section{Results:}

There were no significant regions enhanced by memantine comparing with placebo at any load of $n$-back tests. In contrast, exploring regions reduced by memantine, we found significant reduction of activations within right lingual gyrus and left superior frontal gyrus in comparison between 2-back and 0-back test. A number of correct answers of the 2-back test and time to complete Trail Making Test-A were worse at memantine intervention. Reduced brain activations were associated with worse visuo-spatial working memory caused by memantine.

\section{Conclusions:}

This study reports memantine did not improve visuospatial working memory of the patients with PD-MCl. Treatment of PD should be planned carefully considering the impact for cognitive function. Further study is needed to establish new therapeutic strategy of the patients with PD-MCI.

\section{Introduction}


Parkinson's disease (PD) is a neurodegenerative disease characterized not only by motor symptoms. A cohort study reported that patients with PD had a six-fold greater risk of developing dementia compared with that in normal individuals [1]. A meta-analysis reported that the prevalence of mild cognitive impairment in PD (PD-MCl) was 26\% [2]. In further, Janvin et al. reported that the prevalence was 55\% in a patient group with mean disease duration of over 10 years [3]. Another study reported that $59 \%$ of patients with persistent PD-MCI for 1 year had converted to dementia during the follow-up period [4]. These reports indicate that persistent cognitive impairment of the patients with PD represents increased risk of dementia.

With regards to cognitive impairment in PD, various studies have reported impairments in frontal executive function including working memory [5]. Working memory is responsible for the short-term storage and online manipulation of information necessary for higher cognitive function; impaired working memory can disrupt activities of daily living. One major test to assess working memory is the n-back test, which was developed in the 1950s by Kirchner. Briefly, subjects are presented a sequence of stimuli oneby-one. They must decide and react immediately if the currently presented stimulus is identical to that presented $\mathrm{N}$ trials ago. Many neuroimaging studies have used the verbal $\mathrm{n}$-back task during functional MRI (fMRI) to explore brain activation associated with working memory processing, and n-back task performance is associated with the activations of bilateral frontal and cortical regions [6]. In further, task performance of working memory is associated with dopaminergic neurotransmission in the striatum [7, 8]. Later, a visuospatial $n$-back test was developed in fMRI studies to assess visuospatial working memory in normal subjects $[9,10]$. This test has a merit that the impact of verbal ability is smaller than another cognitive test, and it can assess both visuospatial recognition and working memory in a single test. Recently, we have reported that combinations of functional neuroimaging and the visuospatial nback test are beneficial to evaluate the impaired visuospatial working memory of the patients with PD [11]. Furthermore, fMRI has an advantage to explore medication-related changes of blood-oxygen-leveldependent (BOLD) signal in enhancement or reduction of cortical activations during performing cognitive task. Using $\mathrm{fMRI}$, the pharmacological effect of cholinesterase inhibitors for Alzheimer's disease (AD) [12], or that of a peripheral inhibitor of the enzyme catechol-O-methyltransferase for PD [13], have been studied and detected as significant changes of BOLD signal in comparison with placebo.

Focusing on the pathological features of dementia in PD (PDD) and dementia with Lewy bodies, both are related to the accumulation of diffuse alfa-synuclein aggregates and Alzheimer-type changes [14]. In addition, striatal glutamatergic hyperactivity in an animal model of parkinsonism was reported [15]. Several studies have reported efficacy of cholinesterase inhibitors for PDD [16, 17]. However, cholinesterase inhibitors have an adverse effect of increasing risk of agitation, and these were not more effective than placebo in treating agitation in the patients with $\operatorname{AD}[18,19]$. Another anti-dementia drug for Alzheimer's disease $(A D)$ is memantine ( $\mathrm{N}$-methyl d-aspartate receptor antagonist) which targets glutamatergic neurotransmission. For the treatment of $A D$, meta-analyses have proven that memantine had a better outcome than those receiving placebo, and that memantine was not associated with a significant frequency of adverse events $[20,21]$. There has been also positive effect of memantine for 
PDD, showing that memantine improved global clinical status or behavioral symptoms [22]. However, therapeutic efficacy of memantine for the patients with PD-MCl has not been established.

Therefore, we aimed to investigate whether memantine can alter brain function of the patients with PD$\mathrm{MCl}$, using $\mathrm{fMRI}$. We explored the brain regions associated with visuospatial working memory, and searched the differences in behavioral performance and neuroimaging findings between memantine intervention and placebo. We hypothesized that specific changes of BOLD signals were detected in association with enhancement or reduction of brain activations as a pharmacological influence of memantine.

\section{Materials And Methods}

\section{Participants}

We enrolled 12 right-handed patients with PD-MCl from the Department of Neurology at Nagoya City University Hospital. The study was approved by the local Ethical Committee and complied with national legislation and the Declaration of Helsinki guidelines. All patients provided written informed consent prior to data acquisition. None had any disease except PD affecting motor and cognitive functions. All patients fulfilled the clinical diagnostic criteria according to United Kingdom Parkinson's Disease Brain Bank Criteria for clinical diagnosis [23], and PD-MCI was diagnosed according to the Level II criteria of the Movement Disorder Society Task Force which advocated the detection thresholds of -2 SD had significant impact on the discriminative validity of all measures [24,25]. We excluded patients if they had depression, severe hearing loss, or any other disease that might severely influence data collection. We also excluded patients if they had dementia according to the criteria for PD dementia provided by the Movement Disorder Society Task Force [26]. Two patients withdrew from study. Hence, the population in this study constitutes the 10 patients (eight males and two females) who completed follow-up.

\section{Neuropsychological tests and clinical assessments}

Movement disorder specialists did the complete clinical assessment in all participants. Motor symptoms were assessed with motor section of Unified Parkinson's Disease Rating Scale (UPDRS part3) [27], and daytime sleepiness was assessed with Epworth Sleepiness Scale (ESS) [28]. The dose of dopamine agonists (DA) was normalized using an L-DOPA equivalent daily dose of dopamine agonists (LEDD) [29].

Global cognitive function was assessed with MMSE and Montreal Cognitive Assessment (MoCA). Psychomotor speed and attention were tested with Trail-Making Test Part A (TMT-A) and Paced Auditory Serial Addition Test (PASAT). Executive function and rapid set shifting were tested with Trail-Making Test Part B (TMT-B). Visuospatial function was tested using visuospatial version of 0-back test, and visuospatial working memory was accessed using visuospatial version of 1-back and 2-back test. Using these tests, PD-MCl was classified according to the Level II criteria of the Movement Disorder Society Task Force. 


\section{Visuospatial n-back test}

The n-back test used in this study is a modified version of visuospatial n-back test which was reported in fMRI studies for normal subjects [10]. The details of this test have been reported in our previous paper [11]. In summary, the patients were asked to perform the tests with 3 load levels during the fMRI. The stimuli were white squares randomly presented in 1 of 8 spatial locations on a screen, through a mirror positioned on a head-coil. The presentation of the stimuli was controlled by a program (Presentation software) that initiated the acquisition of the MRI and the behavioural data. For the 0-back test, the subjects were instructed to press the left button with their index finger when a white square was presented. For the 1-back test, they were instructed to press the left button whenever a stimulus was presented in the same location as the previous stimulus. For the 2-back test, they had to press the left button whenever a stimulus was presented in the same location as the two trials previous. When the stimulus was presented in any other location during the 1-back and 2-back test, the patients were instructed to press the right button with the middle finger. The higher the number $\mathrm{n}$ requires the higher level of attention and visuospatial working-memory.

\section{Imaging protocol}

To detect brain regions activated by n-back test, we used a block-design protocol, which alternated between test and rest conditions. The details of imaging protocol have been reported in our previous paper [11]. For test conditions, the white square was randomly presented for 2 second in 1 of 8 possible locations on screen; a black screen was presented for 1 second after stimulus presentation. Each test condition consisted of 15 trials over the course of 45 second; each rest condition lasted for 15 second. During a single scan, each test condition was repeated 4 times, in numerical order (0-1-2 back). Thus, each condition included 60 trials (Fig 1 ). The test was performed during the 'On' state to avoid cognitive change or anxiety arising from being in the 'Off' state during testing.

The figure shows the protocol of visuospatial n-back test. In task conditions, the white square was presented for $2 \mathrm{~s}$ at random in 1 of 8 possible locations on screen, and black screen was presented for $1 \mathrm{~s}$ after the presentation of stimuli. In the 0-back test, the subjects were instructed to press the left button with their index finger when the white square was presented in predetermined locations. They were instructed to press the right button when the stimulus appeared in any other location. In the 1-back test, the subjects were instructed to press the left button when the stimulus presented in the same location as the previous one. In the 2-back test, they were instructed to press the left button whenever a stimulus was presented in the same location as the two trials previous. When the stimulus was presented in any other location during the 1-back and 2-back test, the patients were instructed to press the right button with the middle finger. The details of visuospatial n-back test have been reported in our previous paper [11].

\section{Study design}

The study followed a randomized double-blind crossover design. After inclusion evaluation, patients were randomly assigned to either memantine or placebo during the first study period. Patients in the 
memantine group were given memantine at $5 \mathrm{mg} /$ day in the first week, and the dose was increased by $5 \mathrm{mg} /$ day per week, with the final dose of $20 \mathrm{mg} /$ day from the fourth week to the sixth week. The patients in the placebo group were given a placebo following the same regimen. After the first study period, they underwent 4 weeks washout period. Next, a crossover protocol was performed in the second study period, in which patients given memantine in the first study period were now given placebo. During maximum dose administration in each period, the patients were assessed using fMRI and neuropsychological tests. All subjects were requested to retain a stable medication regime till the end of study period, and they were unaware of the protocol assignment until the end of study (Fig 2).

The figure shows study design. After inclusion, patients were randomly assigned to either memantine or placebo during the first study period. After the first study period, they underwent 4 weeks washout period. Next, a crossover protocol was used in the second study period, in which patients given memantine in the first study period were now given placebo.

\section{Image data acquisition and analysis}

The methods of image data acquisition and analysis have also been described in our previous report [11]. All MRI were acquired with a Siemens Skyra syngo MR E11 3.0 T scanner (Siemens, Germany). Highresolution T1-weighted images were acquired via volumetric 3D spoiled gradient recall sequence. Acquisition parameters were as follows: repetition time $(T R)=1900 \mathrm{~ms}$, echo time $(T E)=2.43 \mathrm{~ms}$, flip angle $(F A)=9$, field of view $(F O V)=256 \times 256 \mathrm{~mm}$, slice thickness $=1 \mathrm{~mm}$, slice gap $=0$, voxel size $=1 \times$ $1 \times 1 \mathrm{~mm}$, number of slices $=176$. The fMRI measurements were performed using a gradient echo EPI sequence: repetition time $(T R)=2500 \mathrm{~ms}$, echo time $(T E)=30 \mathrm{~ms}$, slice thickness $=3 \mathrm{~mm}$, total 149 volumes, with matrix size of $64 \times 64$ and field of view of $192 \times 192 \mathrm{~mm}$, resulting in voxel size of $3 \times 3 \times 3$ $\mathrm{mm}$. All the images were pre-processed and analysed with Matlab (version 8.1, The Mathworks Inc, Natick, MA) and SPM8 software (Department of Cognitive Neurology, London). Images were realigned to correct for movement and normalised to Montreal Neurologic Institute (MNI) space. The transformed image data were smoothed with a Gaussian philtre (full width at half-maximum $=10 \mathrm{~mm}$ ).

The image data were analysed with a random effect procedure and a parametric model to identify the brain areas where the activation correlated with the task. We specified the first level analysis model, estimated and defined the parameters and t-contrasts for n-back test conditions vs. the rest condition. The resulting contrast maps from each contrast and for each subject were then used in a second level random effects analysis for between groups effect (memantine vs. placebo). Group comparison was performed for three loads of the $n$-back test condition. The statistical significance was determined at $\mathrm{P}<0.01$ (uncorrected) with cluster size $>50$ voxels in analysis.

\section{Statistical analysis}

To examine differences in clinical and neuropsychological data resulting from memantine intervention and placebo, each data was compared using the independent t-tests as appropriate. The scores and reaction times of $n$-back test were analysed using one-way analysis of variance, because each load level 
required several neuropsychological cognitive domains. SPSS version 15.0 was used for statistical analyzes. $P<0.05$ was considered significant.

\section{Results}

\section{Clinical and neuropsychological data of the patients with PD-MCI who completed follow-up}

The clinical profiles and demographic data at the time of inclusion were summarised in Table 1. The table shows baseline data of each patient who completed the study. Mean age was $69.0 \pm 2.7$ years, duration from disease onset was $5.1 \pm 2.9$ years, UPDRS part 3 score was $17.0 \pm 6.6$, and MMSE was $26.5 \pm 1.6$. Nine patients received L-DOPA therapy; Four patients were treated with only L-DOPA, and six patients were treated with both DA and L-DOPA.

Table 1. Clinical profiles of the patients who completed follow-up 
$\begin{array}{llll}\text { Age } & \text { Duration } & \text { UPDRS part3 L-DOPA (mg) LEDD }\end{array}$

(years) (years)

(mg)

\begin{tabular}{|c|c|c|c|c|c|}
\hline Patient 1 & 68 & 9 & 31 & 350 & 0 \\
\hline Patient 2 & 72 & 3 & 20 & 250 & 0 \\
\hline Patient 3 & 69 & 2 & 8 & 100 & 100 \\
\hline Patient 4 & 66 & 2 & 19 & 200 & 0 \\
\hline Patient 5 & 72 & 2 & 18 & 200 & 0 \\
\hline Patient 6 & 66 & 4 & 19 & 300 & 75 \\
\hline Patient 7 & 69 & 7 & 17 & 400 & 75 \\
\hline Patient 8 & 74 & 8 & 8 & 150 & 50 \\
\hline Patient 9 & 68 & 9 & 17 & 200 & 150 \\
\hline Patient 10 & 67 & 5 & 13 & 0 & 135 \\
\hline \multirow[t]{2}{*}{ Mean \pm SD } & $69.0 \pm 2.7$ & $5.1 \pm 2.9$ & $17.0 \pm 6.6$ & $215.0 \pm 118.0$ & $58.5 \pm 58.0$ \\
\hline & MMSE & MoCA & PASAT & TMT-A (sec) & TMT-B (sec) \\
\hline Patient 1 & 25 & 20 & 21 & 79 & 420 \\
\hline Patient 2 & 29 & 24 & 28 & 69.7 & 238 \\
\hline Patient 3 & 27 & 25 & 28 & 39.5 & 159 \\
\hline Patient 4 & 28 & 21 & 19 & 48.4 & 151.4 \\
\hline Patient 5 & 29 & 23 & 32 & 77.4 & 194.4 \\
\hline Patient 6 & 26 & 17 & 18 & 100.6 & 379.8 \\
\hline Patient 7 & 25 & 18 & 18 & 65.4 & 420 \\
\hline Patient 8 & 26 & 19 & 16 & 53.6 & 107 \\
\hline Patient 9 & 25 & 18 & 8 & 56.8 & 167.3 \\
\hline Patient 10 & 25 & 16 & 26 & 104.7 & 357 \\
\hline Mean \pm SD & $26.5 \pm 1.6$ & $20.1 \pm 3.1$ & $21.4 \pm 7.1$ & $69.5 \pm 21.4$ & $259.4 \pm 121.9$ \\
\hline
\end{tabular}


Duration: Disease duration; UPDRS part3: motor sections of United Parkinson's Disease Rating Scale; LDOPA: dosage of L-DOPA; LEDD: L-dopa equivalent daily dose of dopamine agonist; MoCA, Montreal Cognitive Assessment; PASAT, Paced Auditory Serial Addition Test; TMT-A: time to complete part A of the Trail Making Test; TMT-B: time to complete part B of the Trail Making Test; sec: seconds

\section{Clinical and neuropsychological data at memantine intervention and placebo}

The clinical data resulting from memantine intervention and placebo were summarized in Table 2. All patients who completed follow-up, used memantine which dose was increased up to $20 \mathrm{mg}$ per day. There was no significant difference in the scores of UPDRS part3 between memantine and placebo. Similarly, there were no significant differences in total scores of MMSE and MoCA, and there were no differences in the score of PASAT and time to complete TMT-B. However, the time to complete TMT-A was significantly longer at memantine intervention comparing with that of placebo $(P<0.05)$. In addition, we found a trend that ESS at memantine intervention was higher than that of placebo $(P=0.07)$.

In comparisons for $n$-back test performance, there were no differences in reaction time. There were no significant differences in the number of correct answers of 0-back and 1-back tests. However, number of correct answers of 2-back test at memantine intervention was significantly fewer than that of placebo (memantine, $10.2 \pm 3.9$; placebo, $12.4 \pm 4.6 ; \mathrm{P}<0.05$ ).

Table 2. Clinical and neuropsychological data at memantine intervention and placebo

\begin{tabular}{llll} 
& Placebo & Memantine & P value \\
\hline UPDRS part3 & $17.0 \pm 6.6$ & $18.0 \pm 5.2$ & N.S. \\
\hline ESS & $7.1 \pm 3.8$ & $8.9 \pm 5.0$ & $\mathrm{P}=0.07$ \\
\hline MMSE & $26.1 \pm 2.2$ & $25.4 \pm 2.5$ & N.S. \\
\hline MoCA & $20.1 \pm 3.1$ & $18.7 \pm 4.1$ & N.S. \\
\hline PASAT & $21.4 \pm 7.1$ & $19.8 \pm 6.7$ & N.S. \\
\hline TMT-A & $69.5 \pm 21.4$ & $86.6 \pm 38.5$ & P $<0.05$ \\
\hline TMT-B & $259.4 \pm 121.9$ & $312.9 \pm 132.6$ & N.S. \\
\hline 0-back test (number) & $17.1 \pm 2.3$ & $16.3 \pm 4.1$ & N.S. \\
\hline 1-back test (number) & $14.9 \pm 3.4$ & $13.9 \pm 2.9$ & N.S. \\
\hline 2-back test (number) & $12.4 \pm 4.6$ & $10.2 \pm 3.9$ & P $<0.05$ \\
\hline 0-back test (seconds) & $790.3 \pm 155.4$ & $800.6 \pm 179.1$ & N.S. \\
\hline 1-back test (seconds) & $991.7 \pm 171.5$ & $1015.4 \pm 208.7$ & N.S. \\
\hline 2-back test (seconds) & $1087.4 \pm 208.7$ & $1167.9 \pm 274.0$ & N.S.
\end{tabular}


This table shows the comparison of neuropsychological findings between memantine intervention and placebo. ESS: Epworth Sleepiness Score; 0-back test (number): number of correct answers in 0-back test (minimum 0, maximum 20); 1-back test (number): number of correct answers in 1-back test (minimum 0 , maximum 20); 2-back test (number): number of correct answers in 2-back test (minimum 0, maximum 20); 0-back test (seconds): reaction time of 0-back test; 1-back test (seconds): reaction time of 1-back test; 2-back test (seconds): reaction time of 2-back test; N.S.: not significant.

\section{fMRI data}

$\mathrm{fMRI}$ analyses were performed to reveal an impact of memantine for the patients with PD-MCl, comparing BOLD signal which associated with visuospatial n-back test. The brain regions shown in Fig 3 were volumes where the t-contrast for decrease and increase between memantine and placebo. There were no significant regions enhanced by memantine compared with placebo at any load of the n-back tests. In contrast, when exploring the regions reduced by memantine intervention comparing with placebo, comparison between the 2-back versus 0-back test revealed significantly reduced activations within right lingual gyrus (LG) and left superior frontal gyrus (SFG). The result of 2-back test showed significantly reduced activations within right superior temporal gyrus and left SFG during memantine intervention, while we did not find any specific findings within frontotemporal or parietal lobes in the 0-back test, 1back test, and comparison between the 1-back versus 0-back test.

The figure shows the results of $\mathrm{fMRI}$ analyses comparing memantine and placebo. The colour-bar represents $t$ values as reference. All the images presented at $P<0.01$ (uncorrected) with cluster size $>50$ voxels in analysis. (A) The coloured regions show change of brain activation during memantine intervention compared with placebo in the 2-back test. We found significantly reduced activation within right superior temporal gyrus and left superior frontal gyrus (SFG). (B) The image shows significantly reduced activation within right lingual gyrus and left SFG during memantine intervention in comparison between the 2-back versus 0-back test. (C) The image shows the enhanced regions during memantine intervention compared with placebo in the 2-back test. We did not find any specific findings in frontotemporal or parietal lobes.

\section{Discussion}

To our knowledge, neuroimaging study to explore changes in brain activation associated with memantine anti-dementia therapy in the patients with PD-MCl has never been done. The present study is the first pilot study that aimed to investigate whether memantine can alter brain function concerning visuospatial working memory of the patients with PD-MCl. Using a combination of visuospatial n-back test and fMRI, we revealed that memantine made local brain function within right LG and left SFG worse, comparing with placebo. In contrast, we did not find specific findings enhanced by memantine.

\section{Memantine reduce brain activations associated with working memory in superior frontal gyrus and lingual gyrus}


There were no significant regions enhanced by memantine compared with placebo at any load of the $n$ back tests. In contrast, fMRI findings of the 2-back versus 0-back test at memantine intervention showed reduction of brain activations in right LG and left SFG. While we did not find any specific regions in the results of 0-back, 1-back, and 1-back versus 0-back test. In behavioral analysis, the number of correct answers of 2-back test during memantine intervention was fewer than that of placebo.

Generally, the changes of task-related brain activation were most prominent for the 2-back condition; subtracting the activated regions in the 0 -back test (the easiest condition) from those in the 2-back test is adequate for the purpose of searching brain regions associated with working memory. The subtracting condition of the 0 -back test from those of the 1-back test had limited statistical power to detect brain areas associated with working memory because the 1-back test requires low-grade visuospatial working memory. Considering these, the deactivation within right LG and left SFG of the patients with PD-MCl represents the impaired visuospatial working memory at memantine intervention.

Concerning the observed regions, it was reported that impaired visual memory was related to either damage to the region or disconnections between the LG and other brain structures [30]. Mangun et al. reported that activation of the $L G$ has been shown in association with selective visual spatial attention in their fMRI study [31], and Machielsen et al. reported that LG has been linked to encoding of complex images [32]. Therefore, deactivation of the $L G$ in the present study may be related to worse visuo-spatial working memory of the patients with PD-MCl during memantine intervention, compared with placebo.

Second, concerning the role of SFG, Carlson et al. reported that this region is dependent on the memory load of visuospatial n-back task in fMRI [10]. In their study which targeted for normal volunteers, comparison of the 2-back versus 0-back tasks revealed bilateral activation in the medial frontal gyrus, superior frontal sulcus, and SFG. They also reported that comparison between the 1-back versus 0-back tasks showed activation only in a few brain areas. However, unlike their result, there was no significant difference in the activation within medial frontal gyrus, comparing between the 2-back versus 0-back test in our study. We considered that fMRI findings associated with working memory were likely to be influenced by daytime sleepiness of the patients with PD-MCI.

In neuropsychological findings, the correct answer rate of 2-back test at memantine intervention was worse than that of placebo. In addition, although it did not reach statistical significance, there was a trend that the score of ESS at memantine was higher than placebo, suggesting that memantine may worsen daytime sleepiness. Focusing on the association between sleepiness and working memory, several fMRI studies have reported that sleepiness decreased performance of working memory [33, 34]. Interestingly, Li et al. reported that functional connectivity between the superior parietal lobule and pre-motor cortex, supplementary motor cortex, and left dorsolateral prefrontal cortex was observed in the primary insomnia group and the control group using resting state fMRI, and they reported that connectivity in the primary insomnia group was weaker between the superior parietal lobule and right superior frontal gyrus (dorsolateral prefrontal cortex) [35]. Considering these, memantine is likely to worsen sleepiness during visuo-spatial n-back task, and it may result in a reduced activation of the SFG. 


\section{Clinical implications for the treatment of PD-MCI}

The development of dementia in the patients with PD is caused by dual pathology, that is, Diffuse Lewy body and AD pathology [14]. First, considering the Diffuse Lewy body pathology, therapeutic choice for cognitive impairment in PD is L-DOPA and DA, because dopamine may improve the neuro-transmission of cortico-striatal circuit. Some studies have reported efficacy of L-DOPA or DA for cognitive impairments of the patients with PD [36,37]. Next, concerning the AD pathology, several studies have reported efficacy of cholinesterase inhibitors for PDD [16, 17], and memantine for PDD [22]. However, neuropathological research for PD-MCl is limited, and therapeutic evidence of PD-MCl has unestablished other than cholinesterase inhibitors.

Focusing on the cognitive influence of memantine for the normal elderly, a double-blind pilot study which targeted for the participants of age-associated memory impairment (no dementia or $\mathrm{MCl}$ ), reported positive effect, suggesting that memantine has a possibility to improve attentional processes if the memory impairment is in subtle stage [38]. Whereas, in the present study which targeted for PD-MCl, we found a negative influence. A time to complete TMT-A and number of correct answers of the 2-back test were worse at memantine intervention. We considered that those results might be caused by both heterogenous pathology of PD-MCl and increase of daytime sleepiness.

It was well known that dopamine agonists tend to increase daytime sleepiness and the risk of sleep attacks for the patients with PD [39]. In the present study, all patients received maximum dose of memantine on study period, and six of ten patients were treated with both dopamine agonists and LDOPA. Therefore, it was plausible that combination of dopamine agonists and maximum dose of memantine might enhance daytime sleepiness which resulted in reduction of psychomotor speed and attention. A recent review which studies the efficacy of anti-dementia drug for PDD, reported that cholinesterase inhibitors improved cognitive function but memantine did not [40]. This study focusing on PD-MCl revealed that memantine did not improve visuospatial working memory and brain activations. The results of this study suggested the importance of careful consideration of daytime sleepiness and/or cognitive function for the treatment of PD. Considering these, maximum dose of memantine is not beneficial for the patients with PD-MCl.

\section{Limitations and strengths}

This fMRI study has some limitations. First, the main limitation was the small sample size. The statistical power to search differences in behavioral performance and neuroimaging findings was restricted. As for the smoothing kernel in $\mathrm{fMRI}$ analysis, although there is no easy answer regarding how much we should smooth imaging data, the main downside to higher smoothing is the loss of spatial specificity. Because of the small sample size, we had to reduce noise as much as we can using relatively high smoothing kernel compared with voxel dimension. Therefore, we used the $10 \mathrm{~mm}$ smoothing kernel instead of the reduction of spatial resolution. Because of these reasons, we could not find significant voxels in imaging analysis with the family-wise error correction. Second, the results of n-back test were likely to be influenced by variety of patients' profile such as the doses of L-DOPA and LEDD of DA. We cannot rule 
out the possibility that variety of treatment may have had differing impacts on behavioural performance. Third, there is also another possibility that neuroimaging results of $n$-back tests were affected by negative emotion including anxiety and depression, because negative emotion has a particularly worsen selectivity of attention as well as motivating action and behavior. To reduce the difference of negative emotion in each patient, we performed fMRI scanning during the 'On' state, because it was predicted that anxiety arising from being in the 'Off' state might influence more compared with 'On' state. However, there is no specific way to quantify real influence of emotions during cognitive task, and it may be a fundamental technical limitation of the activation study using fMRI.

However, the present study has some strengths and novel aspects. First, this is the first neuroimaging study which identified the changes of brain activation associated with memantine for the patients with PD-MCI. Second, we stress that $\mathrm{fMRI}$ is useful to explore the pharmacological influence for central nervous system noninvasively. To clarify association between another therapy for the patients with PD and therapeutic efficacy, further study is needed to be targeted for larger population.

\section{Conclusions}

We have revealed that memantine reduced functional brain activation in right LG and left SFG of the patients with PD-MCl. Memantine did not improve working memory of the patients with PD-MCl. Treatment of PD should be planned carefully considering the impact of medication for cognitive function. Further study is needed to establish new therapeutic strategy for cognitive impairment of the patients with PD-MCl.

\section{Abbreviations}

$\mathrm{fMRI}$, functional magnetic resonance imaging; PD, Parkinson disease; PDD, PD with dementia; PD-MCl, PD with mild cognitive impairment; AD, Alzheimer's disease; BOLD, blood-oxygen-level-dependent; UPDRS, Unified Parkinson's Disease Rating Scale; DA, dopamine agonists; LEDD, L-DOPA equivalent daily dose of dopamine agonists; MoCA, Montreal Cognitive Assessment; TMT-A, Trail-Making Test Part A; TMT-B, TrailMaking Test Part B; PASAT, Paced Auditory Serial Addition Test; AVLT, Auditory Verbal Learning Test; SFG, superior frontal gyrus; LG, lingual gyrus

\section{Declarations}

\section{Acknowledgements}

We wish to thank Satoshi Tanaka for technical support of the design for this neuroimaging study, Ryouhei Akanabe for assistance with $\mathrm{fMRI}$ analysis. We are also grateful to all patients and collaborators who took part in this study.

\section{Authors' contributions}


SK: drafting the manuscript, writing; study concept and design; acquisition and interpretation of data.

NM: study concept and design; review and critique. RCIP-Nagoya Study Group: study concept and design; acquisition of data. All authors have approved the manuscript.

\section{Funding}

This study was supported in part by Grants-in-Aid for Scientific Research on Priority Areas (Grant number is $15 \mathrm{~K} 16362)$.

\section{Availability of data and materials}

The datasets used in this study are available from the corresponding author on reasonable request.

\section{Ethics approval and consent to participate}

This study was approved by the Institutional Review Board of Nagoya City University Hospitals. All subjects gave written informed consent. This study was complied with national legislation and the Declaration of Helsinki guidelines.

\section{Consent for publication}

Not applicable.

\section{Competing interests}

The authors declare that they have no competing interests.

\section{References}

1. Aarsland D, Andersen K, Larsen JP, Lolk A, Nielsen H, Kragh-Sørensen P. Risk of dementia in Parkinson's disease: a community-based, prospective study. Neurology 2001;56(6):730-6.

2. Aarsland D, Bronnick K, Williams-Gray C, Weintraub D, Marder K, Kulisevsky J, et al. Mild cognitive impairment in Parkinson disease: a multicenter pooled analysis. Neurology 2010; 75(12):1062-9.

3. Janvin C, Aarsland D, Larsen JP, Hugdahl K. Neuropsychological profile of patients with Parkinson's disease without dementia. Dement Geriatr Cogn Disord. 2003;15(3):126-31.

4. Pedersen KF, Larsen JP, Tysnes O-B, Alves G. Natural course of mild cognitive impairment in Parkinson disease: A 5-year population-based study. Neurology 2017;88(8):767-74.

5. Lee JE, Cho KH, Song SK, Kim HJ, Lee HS, Sohn YH et al. Exploratory analysis of neuropsychological and neuroanatomical correlates of progressive mild cognitive impairment in Parkinson's disease. JNNP. 2013;85(1):7-16.

6. Hui A, Weijiang He, Jingting Wu, Junjun Z, Zhenlan J, Ling Li. A coordinate-based meta-analysis of the $n$-back working memory paradigm using activation likelihood estimation. Brain Cogn. 2019;132(7):1-12. 
7. Landau SM, Lal R, O’Neil JP, Baker S, Jagust WJ. Striatal dopamine and working memory. Cerebral Cortex. 2009;19:445-454.

8. Backman L, Nyberg L, Soveri A, Johansson J, Andersson M, Dahlin E. et al. Effects of workingmemory training on striatal dopamine release. Science 2011;333:718-718.

9. Courtney SM, Petit L, Maisog JM, Ungerleider LG, Haxby JV. An area specialized for spatial working memory in human frontal cortex. Science 1998;279(5355):1347-51.

10. Carlson S, Martinkauppi S, Rama P, Salli E, Korvenoja A, Aronen HJ. Distribution of cortical activation during visuospatial $n$-back tasks as revealed by functional magnetic resonance imaging. Cereb Cortex. 1998;8(8):743-52.

11. Shoji K, Yoko S, Yoshino U, Noriyuki M. Impairment of the visuospatial working memory of the patients with Parkinson's disease. BMC Neurol. 2021;3;21(1):335.

12. McGeown WJ, Shanks MF, Forbes-McKay KE, Waiter GD, Elrick I, Venneri MG et al. Established Donepezil treatment modulates regional brain activation in early Alzheimer's disease. Current Alzheimer Research 2010;7:415-427.

13. Tambasco N, Muti M, Chiarini P, Tarducci R, Caproni S, Castorioto A, et al. Entacapone Reduces Cortical Activation in Parkinson's Disease with Wearing-Off: A f-MRI Study. PLoS One 2014;15;9(5):e96806.

14. Irwin DJ, White MT, Toledo JB, Xie SX, Robinson JL, Van Deerlin V, et al. Neuropathologic substrates of Parkinson disease dementia. Ann Neurol. 2012;72(4):587-98.

15. Starr MS. Glutamate/dopamine D1/D2 balance in the basal ganglia and its relevance to Parkinson' disease. Synapse 1995;19(4):264-93.

16. Rolinski M, Fox C, Maidment I, Mcshane R. Cholinesterase inhibitors for dementia with Lewy bodies, Parkinson's disease dementia and cognitive impairment in Parkinson's disease. Cochrane Database of Systematic Reviews. 2012.

17. Ballard C, Kahn Z, Corbett A. Treatment of Dementia with Lewy Bodies and Parkinson's Disease Dementia. Drugs Aging 2011;28(10):769-77.

18. Robert JH, Edmund J, Clive GB, Peter B, Richard GB, Roger B, et al. Donepezil for the treatment of agitation in Alzheimer's disease. N Engl J Med. 2007;357(14):1382-92.

19. Clive B, Marisa ML, Edmund J, Simon D, Alan S, Alan T, et al. Quetiapine and rivastigmine and cognitive decline in Alzheimer's disease: randomised double blind placebo controlled trial. BMJ 2005;330(7496):874.

20. Reisberg B, Doody R, Stiffler A, Schmitt F, Ferris S, Jorg H, et al. Memantine in moderate-to-severe Alzheimer's disease. N Engl J Med. 2003 3;348(14):1333-41.

21. Shinji M, Taro K, Nakao I. Memantine Monotherapy for Alzheimer's Disease: A Systematic Review and Meta-Analysis. PLoS One 2015;10(4):e0123289.

22. Emre M, Tsolaki M, Bonuccelli U, Destée A, Tolosa E, Kutzelnigg A, et al. Memantine for patients with Parkinson's disease dementia or dementia with Lewy bodies: A randomised, double-blind, placebo- 
controlled trial. Lancet Neurol. 2010;9(10):969-77.

23. Hughes AJ, Ben-Shlomo Y, Daniel SE, Lees AJ. What features improve the accuracy of clinical diagnosis in Parkinson's disease: a clinicopathologic study. Neurology. 1992;42(6):1142-6.

24. Goldman JG, Holden S, Bernard B, Ouyang B, Goetz CG, Stebbins GT. Defining optimal cutoff scores for cognitive impairment using Movement Disorder Society Task Force criteria for mild cognitive impairment in Parkinson's disease. Mov Disord 2013;28(14):1972-9.

25. Litvan I, Goldman JG, Tröster Al, Schmand BA, Weintraub D, Petersen RC, et al. Diagnostic criteria for mild cognitive impairment in Parkinson's disease: Movement Disorder Society Task Force guidelines. Mov Disord. 2012;27(3):349-56.

26. Emre M, Aarsland D, Brown R, Burn D, Duyckaerts C, Mizuno Y, et al. Clinical diagnostic criteria for dementia associated with Parkinson's disease. Mov Disord. 2007;22(12):1689-707; quiz 1837.

27. Fahn S, Elton RI, Members of the UPDRS Development Committee. Unified Parkinson's Disease Rating Scale. In: Fahn S, Marsden CD, Calne DB, Goldstein M, editors. Recent Developments in Parkinsons Disease: Macmillan Health Care Information. 1987;153-63, 293-305.

28. Johns MW. A new method for measuring daytime sleepiness: the Epworth sleepiness scale. Sleep 1991;14(6):540-5.

29. Tomlinson CL, Stowe R, Patel S, Rick C, Gray R, Clarke CE. Systematic review of levodopa dose equivalency reporting in Parkinson's disease. Movement disorders: official journal of the Movement Disorder Society. 2010;25(15):2649-53.

30. Bogousslavsky J, Miklossy J, Deruaz JP, Assal G, Regli F. Lingual and fusiform gyri in visual processing: a clinico-pathologic study of superior altitudinal hemianopia. JNNP 1987; 50(5):607614.

31. Mangun GR, Buonocore MH, Girelli M, Jha AP. ERP and fMRI measures of visual spatial selective attention. Hum Brain Mapp. 1998;6(5-6):383-389.

32. Machielsen WC, Rombouts SA, Barkhof F, Scheltens P, Witter MP. fMRI of visual encoding: Reproducibility of activation. Human Brain Mapping. 2000;9(3):156-164.

33. Nissen C, Kloepfer C, Feige B, Piosczyk H, Spiegelhalder K, Voderholzer U, et al. Sleep-related memory consolidation in primary insomnia. J Sleep Res. 2011;20:129-136.

34. Walker MP: Cognitive consequences of sleep and sleep loss. Sleep Med. 2008;9(Suppl 1):S29-34.

35. Li Y, Wang E, Zhang H, Dou S, Liu L, Tong L, et al. Functional connectivity changes between parietal and prefrontal cortices in primary insomnia patients: evidence from resting-state fMRI. Eur $\mathrm{J}$ Med Res. 2014;10;19(1):32.

36. Masahiro I, Hiroshi K, Satoshi U. Can levodopa prevent cognitive decline in patients with Parkinson's disease? Am J Neurodegener Dis. 2017;15;6(2):9-14.

37. Livia B, Valentina P, Maria CM, Raffaele B, Cesare I, Paolo S. The effect of dopamine agonists on cognitive functions in non-demented early-mild Parkinson's disease patients. Funct Neurol 2013;28(1):13-7. 
38. Ferris S, Schneider L, Farmer M, Kay G, Crook T. A double-blind, placebo-controlled trial of memantine in age-associated memory impairment (memantine in AAMI). Int J Geriatr Psychiatry. 2007;22(5):448-55.

39. Paus S, Brecht HM, Koster J, et al. Sleep attacks, daytime sleepiness, and dopamine agonists in Parkinson's disease. Mov Disord. 2003;18(6):659-667.

40. Wang H-F, Yu J-T, Tang S-W, Jiang T, Tan C-C, Meng X-F, et al. Efficacy and safety of cholinesterase inhibitors and memantine in cognitive impairment in Parkinson's disease, Parkinson's disease dementia, and dementia with Lewy bodies: systematic review with meta-analysis and trial sequential analysis. J Neurol Neurosurg Psychiatry. England; 2015; 86(2): 135-43.

\section{Figures}

\section{\begin{tabular}{|l|l|l|l|}
\hline Initiation & 0back & 1back & $\mathbf{2 b a c k}$ \\
\hline
\end{tabular}}

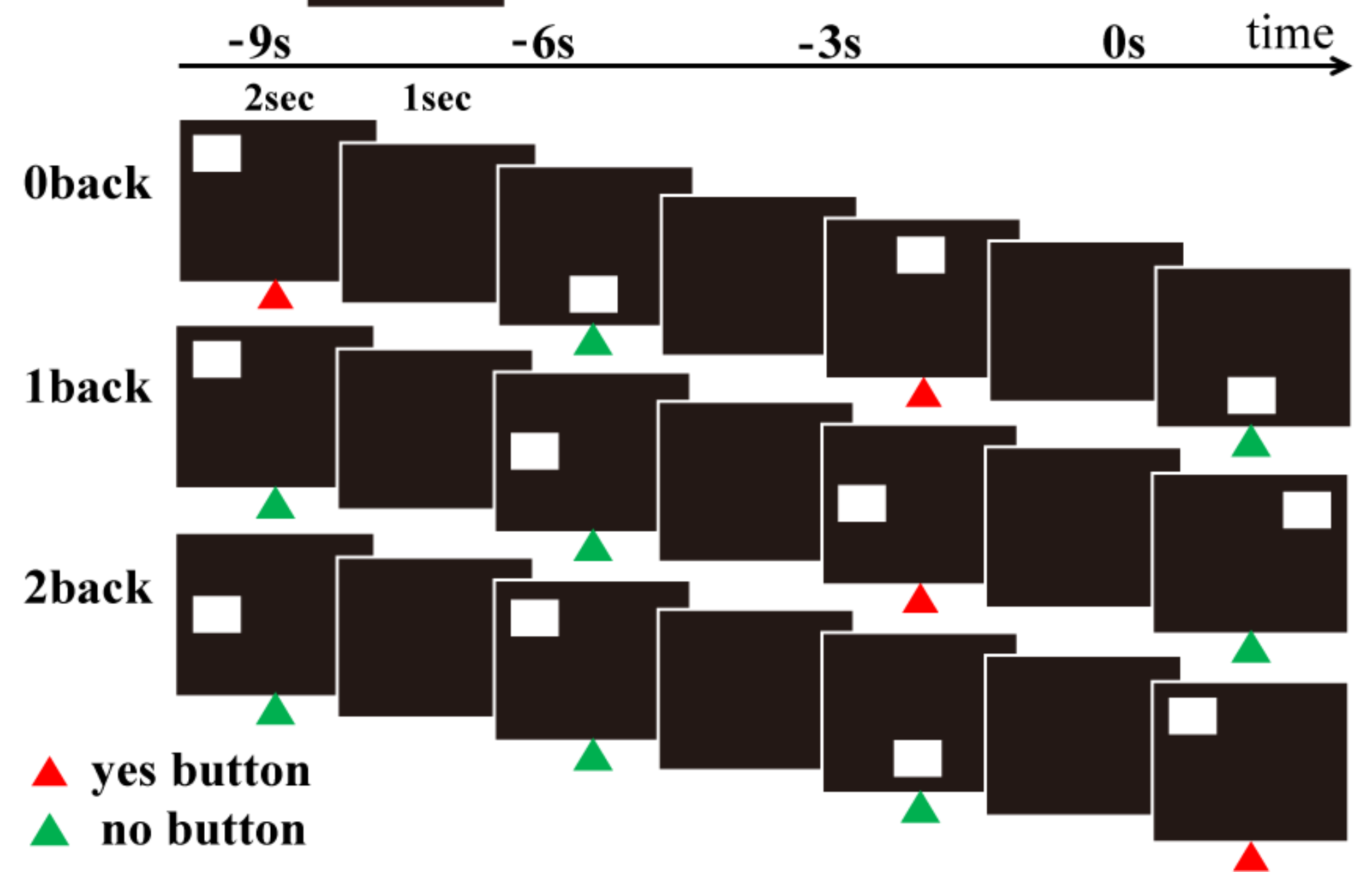

Figure 1 


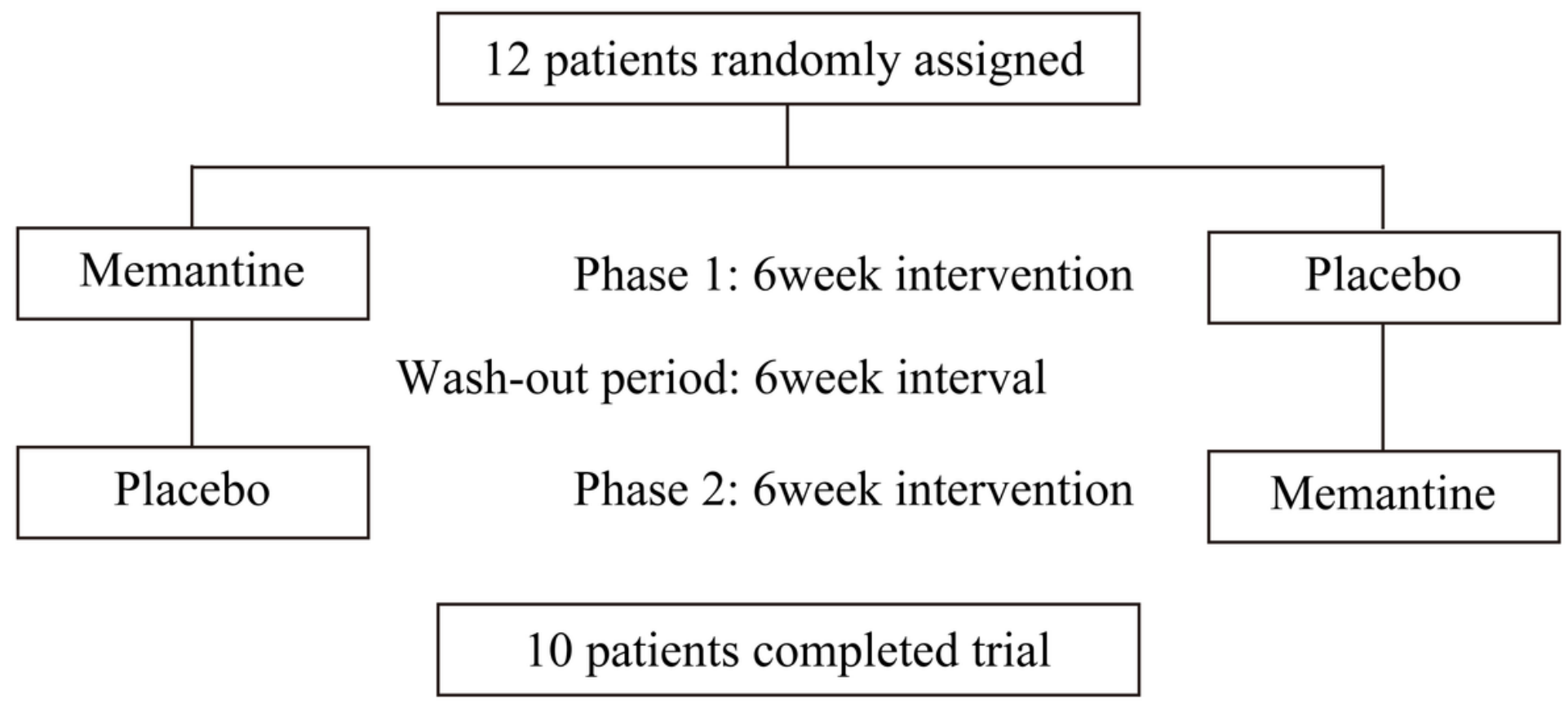

Figure 2

Study design 

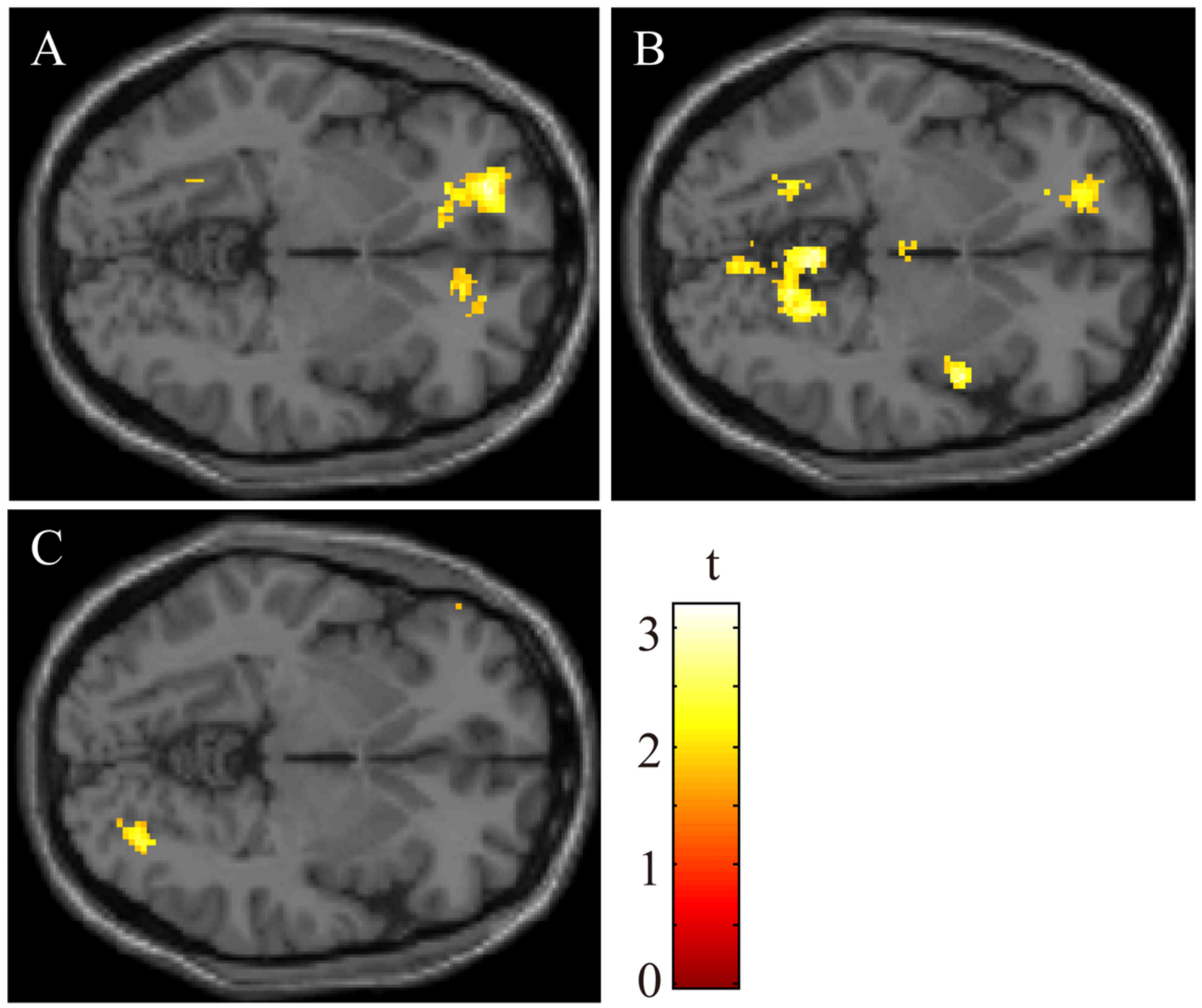

Figure 3

fMRI analyses comparing memantine and placebo 